\title{
Du fait de pousser, diriger et manipuler les gens
}

\section{Eberhard Wolff}

PD Dr rer. soc., rédacteur culture, histoire, société

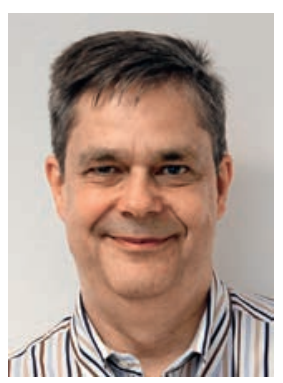

Références

1 Sur YouTube sous "piano stairs".

$213^{\mathrm{e}}$ Congrès Suisse pour l'économie de la santé et les sciences de la santé \& forum d'avenir santé, octobre 2016, www.skgg.ch (présentations et vidéos sur le site)

3 Armellino D, et al. Using High-Technology to Enforce Low-Technology Safety Measures: The Use of Third-party Remote Video Auditing and Realtime Feedback in Healthcare. Clinical Infectious Diseases. 2012;54(1):1-7.

4 Maier E, Reimer U. Soutenir la motivation à changer de comportement grâce au e-nudging. Bull Méd Suisses. 2016;97(43): 1498-1500.

5 Wolff E. Combien de compétences en santé voulons-nous? Bull Méd Suisses. 2009;90(39):1526. 6 Sur YouTube sous «Citibanan» et "Odenplan».
Odenplan, une station de métro centrale à Stockholm, a gagné en célébrité lorsqu'en 2009 on y a installé sur un escalier fixe (à côté d'un escalier roulant) une sorte de clavier qui, à la joie spontanée de nombreux passants, produisait des notes de piano lorsque l'on marchait dessus. Apparemment, deux tiers de passants supplémentaires prenaient désormais l'escalier fixe plutôt que roulant [1] et sautillaient dessus. L'expérience est devenue l'un des exemples les plus populaires du «nudging». Cette technique vise à inciter les gens à adopter un comportement jugé bon, non en les convainquant ou en utilisant des contraintes et interdictions immédiates, mais avec des structures rencontrées (ici, le jeu et le plaisir). L'économie a longtemps basé exclusivement ses modèles sur un Homo oeconomicus agissant de manière rationnelle dans son intérêt personnel. L'économie comportementale plus récente examine cependant un genre de Homo subcorticalis ou de Homo instinctivus, au-delà de la décision rationnelle. Le nudging s'efforce de diriger les modes de comportement humains grâce aux modèles de l'économie comportementale. En fonction de leurs valeurs, les uns appellent cela «influencer», les autres «manipuler». Cela vaut aussi pour les félicitations du smartphone pour le dix-millième pas du jour. Ou pour le repas que la cantine juge sain et qui est particulièrement mis en vedette et proposé moins cher. En matière de médecine préventive et d'objectifs médico-économiques, notamment, le nudging est fréquent. Récemment, une annonce de congrès a pompeusement présenté le nudging comme «Une nouvelle voie vers l'avenir" [2]. On le sait, les voies vers l'avenir peuvent être des boulevards, des autoroutes, mais aussi des pistes pleines de bosses ou même des impasses. Outre la publicité et les descriptions enthousiastes de projets en cours, le congrès a été l'occasion d'entendre un certain scepticisme à l'égard du nudging. Ainsi, le concept serait éclectique et incohérent du point de vue logique. Son efficacité ne serait pas totalement attestée et ne devrait pas être surestimée. Moi-même, je me suis demandé si un tel escalier en touches de piano ne plongerait pas dans un premier temps la gare CFF de Bâle dans le chaos aux heures de pointe. Après quelques semaines, les foules lassées par cette soupe musicale - d'ailleurs susceptible de rendre fous les gens qui travaillent sur place - graviraient les marches quatre à quatre. Et au final, les escaliers roulants de la passerelle seraient peut-être encore plus encombrés.

Un conférencier du congrès a présenté comme exemple de nudging l'étude (controversée) menée avec une caméra au-dessus du lavabo d'une unité de soins intensifs. Sa simple installation a augmenté la fréquence du lavage des mains du personnel médical [3]. Thomas D. Szucs, membre du conseil consultatif du congrès et présentateur, n’a pas été le seul à faire le rapprochement avec le «Big Brother» d'Orwell. Et ce n'est pas un hasard si une intervenante a intitulé son atelier «Faut-il parfois forcer les patients à être heureux?». Parce que l'impulsion peut, presque imperceptiblement, se transformer en contrainte.

Non, je ne veux pas diaboliser le nudging. C'est quelque chose que nous faisons tous, tout le temps. Même ce texte cherche, à travers quelques trucs simples, à vous mettre de mon côté. Mais si le nudging devient tout à coup une grande campagne enseignée, orchestrée pour soutenir l'Etat, nous devons être vigilants.

Un article récemment paru ici expliquait que le nudging augmente la responsabilité personnelle et la participation et répond au besoin d'autonomie des gens en matière de santé [4]. Pour moi, être dirigé de l'extérieur, ce n'est pas se montrer responsable. Et c'est tout le contraire de l'autonomie. Une marionnette ne peut pas non plus participer, puisque c'est un acte délibéré. Enfin, le nudging systématique est la négation de ma liberté de choix conscient, de ma raison et de mon souhait de transparence. En médecine, je vante donc les mérites des ennemis naturels du nudging, tels que le «consentement éclairé» ou le concept de «compétence de santé», tant que l'on entend par là une réflexion indépendante et non simplement une bonne conduite informée [5].

$\mathrm{Au}$ fait, la nouvelle gare "Citibanan» sera inaugurée en août prochain sous la station de métro Odenplan. Elle ne comportera que des escaliers roulants [6]. C'est dommage pour tous ceux qui, comme moi, aiment grimper les marches - de leur propre volonté. Du moins auront-ils la certitude de ne pas être subtilement poussés. 